\title{
Adaptación del Cuestionario de Voluntad de Trabajo a una muestra de universitarios españoles
}

\author{
Ana Andrés ${ }^{1}$, Facundo $\mathrm{Abal}^{2}$, Gabriela Lozzia ${ }^{2}$, Juana Gómez-Benito ${ }^{1 *}$, \\ María E. Aguerri ${ }^{2}$, María S. Galibert ${ }^{2}$ y Horacio Attorresi ${ }^{2}$
}

1 Universidad de Barcelona, España

${ }^{2}$ Universidad de Buenos Aires, Argentina

\begin{abstract}
Resumen: El objetivo del presente estudio es evaluar las propiedades psicométricas del Cuestionario de Voluntad de Trabajo adaptado a una muestra de estudiantes universitarios españoles, tanto en su versión de autoinforme como en la versión destinada a evaluar los pares. Los participantes de este estudio son 194 estudiantes de la Universidad de Barcelona, a los que se aplicaron tanto el cuestionario de Voluntad de Trabajo (CVT) como su versión para evaluar a los pares (CVT-P), así como las subescalas de Perseverancia y Distorsión del Big Factor Questionnaire. Los resultados sugieren la eliminación de dos de los ítems de los cuestionarios CVT y CVT-P. Las correlaciones ítem-total corregidas y la consistencia interna fue adecuada para ambos, y el análisis factorial confirmatorio confirma su unidimensionalidad. La relación entre la escala CVT y las demás medidas criterio es adecuada. En conclusión, podemos afirmar que las escalas CVT y CVT-P obtuvieron unas adecuadas propiedades psicométricas tras la depuración de las mismas. Palabras clave: Voluntad de trabajo; Perseverancia; Adaptación de tests; Propiedades psicométricas.
\end{abstract}

\section{Introducción}

Llevar adelante una carrera de estudios universitarios depende de la concreción de una serie de metas a corto, medio y largo plazo. En consecuencia, una elevada capacidad para autorregular las propias conductas, emociones y cogniciones y la autoimposición de objetivos son aspectos destacados en la personalidad de un estudiante. Sin embargo, siempre existen factores internos y externos a la persona que pueden poner en riesgo la consecución de sus objetivos. En efecto, la determinación de una meta y el compromiso con ésta no brindan un pronóstico certero sobre si la misma podrá ser o no alcanzada.

En el marco de una investigación que estudia atributos de la personalidad relevantes en el ámbito de la educación, Lozzia, Abal, Aguerri, Galibert y Attorresi (2007) definieron la Voluntad de Trabajo (VT) como la tendencia de un individuo a asumir sus responsabilidades, motivado por su propio deseo de realizar un buen trabajo sin rehuir el esfuerzo que ello implica, aún cuando dichas tareas puedan no ser de su agrado. La definición de la VT como un rasgo se sustenta en el hecho de que diversos modelos de personalidad y de motivación han delimitado constructos similares. Como aseguró Moreno-Jiménez (2007), la capacidad de autorregulación para alcanzar objetivos ha sido identificada por numerosos teóricos como una característica diferencial de las personas.

* Dirección para correspondencia [Correspondence address]: Juana Gómez-Benito, Departamento de Metodología de las Ciencias del Comportamiento, Facultad de Psicología, Paseo Valle Hebrón, 171, 08035 Barcelona (España). E-mail: juanagomez@ub.edu
Title: Adaptation of Will-to-Work Questionnaire to a sample of Spanish undergraduate students

Abstract. The aim of the present study was to assess the psychometric properties of the Will-to-work scale both in its self-reported and peer forms in a sample of Spanish undergraduate students. Participants of this study were 194 students from the University of Barcelona, who completed the following questionnaires: Will-to-work scale (WW), peer Will-to-work scale (PWW), and the Perseverance and Distortion subscales from the Big Factor Questionnaire. Results of this study suggested the need of deleting two of the items from the WW and PWW. Adequate item-total correlations were found, as well as acceptable values of internal consistency coefficients in both scales. The confirmatory factor analysis confirmed the one-factor structure of both scales. Relationships between WW and other criteria measures were also adequate. Finally, we can conclude that WW and PWW questionnaires obtained adequate psychometric properties after considering their refinement.

Key words: Will-to-work; Perseverance; Test adaptation; Psychometric properties.

En un estudio comparativo se halló que las formulaciones factorialistas de Guilford (1975), Cattell (Cattell, Eber y Tatsuoka, 1970), Eysenck (Eysenck y Eysenck, 1985), Zuckerman (2002) y el Modelo de los Cinco Grandes (e.g. Caprara, Barbaranelli y Borgogni, 1993; Costa y McCrae, 1999) contenían variables que evaluaban aspectos muy relacionados con la VT (Lozzia et al., 2007). No obstante, cada operacionalización reflejó una definición conceptual diferente en función del énfasis que se le ha otorgado tanto a la capacidad de autocontrol del sujeto como a la energía con que éste realiza sus tareas. El modelo de la personalidad propuesto por Guilford (1975) comprende dos variables relacionadas con la VT. La primera de ellas es la llamada Actividad General, que hace referencia a la energía con la que se llevan a cabo las actividades, contemplando además la constancia con la que se realizan las acciones. La segunda variable es el Retraimiento, que caracteriza aspectos vinculados al autocontrol y la capacidad de realizar un esfuerzo persistente. La VT también se relaciona conceptualmente con el polo negativo del Psicoticismo descrito por Eysenck (Eysenck y Eysenck, 1985) y con una elevada Fuerza de Super-ego en el modelo de Cattell (Cattell et al., 1970). Estos modelos enfatizan la relación del individuo con la sociedad y evalúan la responsabilidad a partir del ajuste a las normas de convivencia. La VT, en cambio, es más acotada describiendo exclusivamente el compromiso que el individuo tiene con el trabajo. En relación con el Modelo de los Cinco Grandes factores de la personalidad se observó que el dominio Escrupulosidad (también llamado Responsabilidad o Tesón según las distintas traducciones del término original Conscientiousness) incluye las características primordiales de la VT. A pesar de que nin- 
guna de las subdimensiones de este dominio resultó estrictamente equivalente a la definición conceptual de VT, se consideró que la faceta Perseverancia (Caprara et al., 1993) era la más próxima. Ambos constructos comparten características como la capacidad de trabajar duramente y la búsqueda activa de logros. Así también, la faceta Dinamismo (perteneciente al dominio Energía) alude tangencialmente a VT en tanto que mide, entre otras características, el entusiasmo que se imprime al realizar las actividades y la capacidad para desarrollar comportamientos vigorosos. Finalmente, desde otro modelo pentafactorial propuesto por Zuckerman (2002), la VT aparece reflejada en la subescala de Esfuerzo de Trabajo (Work Effort). Ésta evalúa la preferencia por los desafíos y el trabajo duro así como la cantidad de energía que es aplicada al trabajo y a otras tareas. Según este modelo, de la energía total con la que cuenta el sujeto para sus actividades, se aísla una parte que puede ser encauzada para cumplir con los objetivos de su trabajo.

La Teoría del Control de la Acción, (Kuhl, 1985, 1994a) también consideró que la capacidad para instrumentar procesos autorregulados depende de diferencias individuales en una característica de la personalidad denominada Orientación estado-acción. Para este autor, en las personas en las que predomina la orientación hacia la acción se observa una mayor eficacia en el rendimiento y una capacidad para sobreponerse a pequeños fracasos. En cambio, las personas que se orientan mayormente al estado suelen detenerse en metas o estados afectivos alternativos a la intención, lo que reduce los recursos cognitivos disponibles para alcanzar la meta. El cuestionario Hakemp-90 (Kuhl, 1994b) permite medir esta orientación volitiva a través de tres dimensiones: a) Iniciativa vs. Vacilación, que evalúa la capacidad del sujeto para aplicar un conjunto de mecanismos que le permitan ejecutar la acción planificada con prontitud y sin vacilaciones; b) Persistencia vs. Volatilidad, que mide la capacidad del individuo para concentrarse en la realización de la tarea durante el tiempo necesario, evitar distracciones y concluir el trabajo; c) Resolución vs. Preocupación, que valora la eficacia del individuo para separarse de pensamientos vinculados a fracasos previos dejando que los mismos no interfieran en la concreción de la tarea.

Características similares a la VT también fueron consideradas por Alonso Tapia, Montero y Huertas (2000) al describir la motivación en personas adultas y su relación con logros académicos y profesionales. Estos autores definieron la Disposición habitual hacia el esfuerzo (DES) y el Desinterés por el trabajo y rechazo del mismo (DT) como variables moduladoras del efecto motivador de las metas. Las mismas constituyen la actitud del individuo hacia los medios usados para alcanzar los logros personales. Ambos constructos pueden medirse en universitarios, junto con otras variables, con el Cuestionario MAPE-3 (Motivación de Aprendizaje y Ejecución-3, Alonso Tapia et al., 2000) y aparecen también en otras versiones adaptadas para adolescentes (Alonso Tapia, 2005; Alonso Tapia y Sánchez Ferrer, 1992; Montero y Alonso Tapia, 1992). La escala DES del MAPE-3 mide la tendencia habitual de un individuo a asumir una sobrecarga de trabajo y a reconocerse como un individuo ocupado que trabaja más que la mayoría de las personas. A diferencia de la escala DES, la VT no se define como una tendencia a sobrecargarse de trabajo sino que describe la capacidad de una persona para responsabilizarse de aquellas tareas que acepta y terminarlas sin dilaciones. Así también, mientras que la DES no implica un gusto particular por el esfuerzo, este aspecto sí es característico de la VT. La escala DT del MAPE-3 describe la tendencia general a afrontar el estudio (o cualquier otro trabajo) sin interés, con poca atención y concentración por suponerlo pesado y aburrido. Consecuentemente, se explica la propensión a evitar o a no concluir la tarea a menos que exista una presión externa para realizarla. Dentro de los aspectos considerados por la DT se incluyen indicadores para la valoración de la persona como vaga tanto desde su propia percepción como la de los demás. Sin embargo, esto no suele ser fácilmente reconocido por los universitarios, quienes tienden a definirse como muy voluntariosos aun cuando sus docentes opinan lo contrario (Lozzia et al., 2008). Las personas con baja VT no necesariamente tienden a rechazar el trabajo en sí mismo, sino que rechazan el esfuerzo que éste implica hasta el punto de especular con recursos alternativos para reducir al mínimo el gasto de energía requerido.

Todas las operacionalizaciones de constructos similares a la VT mencionadas se realizan con cuestionarios extensos o inventarios de amplio espectro que proporcionan una medida global de la personalidad. Actualmente se dispone de un breve cuestionario validado con muestra argentina, que mide específicamente la VT en estudiantes universitarios. La escala presentó una adecuada consistencia interna. Asimismo, el análisis de la estructura interna del cuestionario puso de manifiesto que los ítems de la escala se agrupan bajo una única dimensión que mide los aspectos motivacionales relacionados con la perseverancia y la responsabilidad. Acorde a lo esperado a nivel teórico, fueron verificadas empíricamente las relaciones establecidas con las facetas Perseverancia y Dinamismo del BFQ (Abal, Lozzia, Aguerri, Galibert y Attorresi, 2007). Además de esta versión autoinforme, también se dispone de otra forma compuesta por los mismos ítems que le permite al alumno evaluar la VT de su grupo de pares. De esta manera, la comparación de las puntuaciones obtenidas en ambas versiones cuantifica la distancia percibida por el estudiante entre su VT y la de su grupo de referencia (Abal, Lozzia, Aguerri, Attorresi y Galibert, 2007).

El objetivo del presente estudio es evaluar las propiedades psicométricas de la adaptación del Cuestionario de Voluntad de Trabajo en muestra española tanto en su versión autoinforme (CVT) como en su versión para evaluar a los pares (CVT-P).

\section{Método}

\section{Adaptación del contenido del cuestionario}

Los cuestionarios CVT y CVT-P se crearon específicamente para medir la VT (Lozzia et al., 2007) y fueron valida- 
dos en una muestra de estudiantes universitarios de Psicología de la Universidad de Buenos Aires, Argentina (Abal, Lozzia, Aguerri, Galibert y Attorresi, 2007). Por tanto, previamente a su aplicación a muestra española fue necesario llevar a cabo un análisis del contenido de los mismos. Para ello se contó con la colaboración de dos jueces expertos de la facultad de Psicología de la Universidad de Barcelona, con amplia trayectoria en docencia universitaria. Ambos señalaron la necesidad de reescribir uno de los ítems del cuestionario: "Me dejo estar en el cumplimiento de mis deberes". Las opiniones de los jueces expertos fueron recogidas por los autores del presente trabajo, quienes reformularon el ítem de la siguiente manera: "Suelo dejar para más tarde el cumplimiento de mis obligaciones", considerándose más adecuado para la versión española del test. Esta modificación fue a su vez consensuada con los autores de la versión original, a fin de obtener garantías de la equivalencia lingüística y conceptual de la adaptación de los cuestionarios. La adaptación de los cuestionarios CVT y CVT-P están disponible en los Anexos 1 y 2.

\section{Participantes}

Los participantes del presente estudio son 194 estudiantes de la Universidad de Barcelona. La edad media de los participantes fue de 21.26 años $(D T=4.16$ años), participando un mayor porcentaje de mujeres $(81.4 \%$ ) que de hombres (18.6\%). Prácticamente la mitad de ellos trabajaban a la vez que realizaban sus estudios universitarios (54.1\%). La media de horas semanales de trabajo fue 18. Similares características presentó la muestra argentina (Abal, Lozzia, Aguerri, Attorresi et al., 2007).

\section{Instrumentos}

Los instrumentos aplicados en este estudio fueron los CVT y CVT-P, así como las subescalas de Perseverancia y Distorsión del cuestionario Big Factor Questionnaire en su versión en castellano (BFQ, Caprara et al, 1993; adaptación de Bermúdez, 1995). El protocolo también indagó sobre datos personales como edad, sexo, condición laboral e incluyó un ítem de respuesta cerrada sobre el autoconcepto respecto de la voluntad de trabajo formulado como: "Se considera a sí misma como una persona con poca, mediana o mucha voluntad de trabajo".

El CVT (Anexo 1) consta de 16 ítems con siete opciones de respuesta tipo Likert (nunca, casi nunca, pocas veces, a veces, con frecuencia, casi siempre y siempre). Por ende, el rango de puntuaciones posibles es de 16 a 112 puntos. En un estudio previo, se aplicó el cuestionario de VT a una muestra de estudiantes de Buenos Aires (Argentina), obteniendo una alfa de Cronbach de 86 (Abal, Lozzia, Aguerri, Attorresi et al., 2007).

El CVT-P (Anexo 2) posee el mismo formato y contenido que la escala anterior, pero se administra para que la persona evalúe a terceras personas. En este caso, los participantes evalúan a sus compañeros de clase o pares. El alfa de Cronbach obtenido tras su aplicación a estudiantes de Bue- nos Aires fue de .89. (Abal, Lozzia, Aguerri, Attorresi et al., 2007).

Finalmente, los inventarios de Perseverancia y Distorsión son dos subescalas pertenecientes al BFQ. Cada una de estas escalas está formada por 12 ítems que se contestan en una escala Likert de 5 opciones de respuesta (desde Completamente verdadero para mí a Completamente falso para mí). La escala de Perseverancia evalúa la persistencia y la tenacidad con que se llevan a cabo las tareas y actividades emprendidas. Consecuentemente, esta variable está directamente relacionada con la medida de VT. La escala de Distorsión tiene por objetivo identificar los perfiles falseados, puesto que aquellos sujetos motivados por dar de sí una imagen favorable pueden distorsionar sus resultados. Por tanto, esta escala permite evaluar la tendencia a alterar las respuestas con el propósito de ofrecer una falsa impresión de uno mismo. Las puntuaciones altas en esta subescala indicarán una disposición a mostrar una imagen de uno mismo artificiosamente positiva. En el manual de la adaptación, Bermúdez (1995) refiere un índice de consistencia interna alfa de Cronbach de .76 para la subescala de Perseverancia y de .77 para Distorsión. En la presente investigación se hallaron índices ligeramente más bajos (alfa de Cronbach de .70 para cada una de las medidas). El análisis factorial exploratorio pone de manifiesto que el BFQ está formado por cinco factores, obteniéndose un porcentaje de varianza explicada del 57\% y adecuadas cargas factoriales (Bermúdez, 1995). Asimismo, el manual del test apunta a la adecuada validez convergente de la escala, basada en las correlaciones significativas con otras medidas de personalidad.

\section{Procedimiento}

Los cuestionarios fueron aplicados a alumnos de segundo curso de Psicología de la Universidad de Barcelona. Las respuestas dadas por los alumnos fueron voluntarias y anónimas, respetándose en todo momento la confidencialidad de los datos. La administración se hizo en clase y de forma autoadministrada. Cada uno de los participantes respondió a todos los cuestionarios mencionados anteriormente. Cabe destacar que los alumnos respondieron el CVT-P evaluando la voluntad de trabajo que consideran que muestran sus compañeros de clase como grupo.

\section{Análisis de datos}

Los análisis presentados en el presente trabajo se llevaron a cabo mediante el programa PASW Statistics 17. Asimismo, se utilizó el programa AMOS 17.0 para realizar el análisis factorial confirmatorio (AFC).

Con el objetivo de analizar la posible influencia de determinadas variables sociodemográficas en la VT de los participantes de este estudio, se aplicó la correlación de Pearson, así como la prueba $t$ de Student de comparación de medias.

Las correlaciones ítem-total corregidas y los índices alpha de Cronbach se interpretaron de acuerdo con los criterios 
propuestos por Nunnally y Bernstein (1994). Antes de llevar a cabo el AFC se analizó la normalidad multivariante de los datos mediante el índice de Mardia (1970). De acuerdo con Bentler (2005), valores por encima de 5.0 para el índice normalizado de Mardia son indicadores de no normalidad multivariante. En consecuencia se aplicó el método de estimación de mínimos cuadrados no ponderados, obteniéndose los siguientes índices de bondad de ajuste: prueba de chi cuadrado, GFI (goodness-of-fit index; Jöreskog y Sörbom, 1986), AGFI (adjusted goodness-of-fit index; Jöreskog y Sörbom, 1986), NFI (normed fit index; Bentler y Bonet, 1980), y SRMR (standardised root mean square residual; Jöreskog y Sörbom, 1986). Los puntos de corte utilizados para determinar un ajuste adecuado fueron: igual o superior a .90 para GFI y AGFI, igual o superior a .60 para NFI e igual o inferior a .08 para SRMR (Hu y Bentler, 1999).

Finalmente, el análisis de la relación entre el cuestionario CVT y otras variables fue analizado mediante correlaciones (Spearman o Pearson en función del tipo de variable).

\section{Resultados}

\section{Análisis de la consistencia interna}

Los índices de discriminación se basaron en la correlación ítem-total corregida. Prácticamente todos los elementos obtuvieron valores adecuados a excepción de dos de ellos. Los ítems 4 "Prefiero dedicarme a varias actividades que a pocas" y 13 "Prefiero saber un poco de distintos temas que mucho de algunos po$\cos ^{\prime}$ no discriminaron satisfactoriamente entre los estudiantes con alta y baja puntuación en VT, obteniendo índices de -.08 y .13 , respectivamente.

Los índices de discriminación del CVT-P dieron lugar a resultados similares a los encontrados en la escala VT. La mayor parte de los ítems obtuvieron correlaciones ítem-total corregidas superiores a .40. Nuevamente, los ítems 4 y 13 presentaron poco poder discriminante, ya que obtuvieron valores de -.14 y .16 , respectivamente.

Estos resultados sugieren la eliminación de los ítems 4 y 13 tanto del CVT como del CVT-P, puesto que no discriminan adecuadamente en base al nivel de voluntad de trabajo presentada por los participantes. La tabla 1 muestra los valores alcanzados por los restantes ítems.

El coeficiente alpha de Cronbach obtenido en el CVT fue de .88 , valor que puede considerarse adecuado. Un resultado similar se halló en relación a la consistencia interna de la escala de CVT-P, alcanzando un alfa de Cronbach de .92. En la Tabla 1 se muestra la consistencia interna que alcanzaría el test en caso de eliminar cada uno de los ítems. Como se puede observar, todos los ítems contribuyen a la consistencia interna tanto del CVT como del CVT-P y en consecuencia no es necesario plantear la posibilidad de eliminar ningún otro ítem.

\section{Dimensionalidad del test}

Considerando que la voluntad de trabajo ha sido definida como un rasgo unitario, se puso a prueba un modelo unifactorial para ambos cuestionarios mediante un AFC. Los CVT y CVT-P deberían tener la misma estructura, puesto que están midiendo el mismo constructo, ya sea referida a uno mismo o a los compañeros de clase.

Tabla 1. Índices de discriminación y consistencia interna en caso de eliminar ítems de los CVT y CVT-P.

\begin{tabular}{lcccc}
\hline \multicolumn{3}{c}{ Items } & \multicolumn{2}{c}{ CVT } \\
\multicolumn{2}{c}{ Discriminación } & $\begin{array}{c}\text { Consistencia } \\
\text { interna }\end{array}$ & Discriminación & $\begin{array}{c}\text { Consistencia } \\
\text { interna }\end{array}$ \\
\hline VT1 & .398 & .881 & .440 & .923 \\
VT2 & .537 & .876 & .620 & .917 \\
VT3 & .610 & .872 & .709 & .914 \\
VT5 & .542 & .875 & .723 & .913 \\
VT6 & .575 & .874 & .603 & .918 \\
VT7 & .598 & .873 & .482 & .921 \\
VT8 & .619 & .872 & .650 & .916 \\
VT9 & .618 & .872 & .683 & .915 \\
VT10 & .647 & .871 & .668 & .916 \\
VT11 & .626 & .871 & .749 & .913 \\
VT12 & .626 & .871 & .648 & .916 \\
VT14 & .453 & .879 & .749 & .913 \\
VT15 & .714 & .868 & .755 & .913 \\
VT16 & .358 & .891 & .623 & .918 \\
\hline
\end{tabular}

Las pruebas de KMO y de esfericidad de Bartlett pusieron de manifiesto la adecuación de los datos para realizar la reducción de los datos, tanto en el CVT $\left(\mathrm{KMO}=.91, \chi^{2}(91\right.$, $n=194)=1009.63, p<.001)$ como en el CVT-P $(\mathrm{KMO}=$ $\left..92, \chi^{2}(91, n=194)=1479.93, p<.0001\right)$. Puesto que los datos obtenidos no mostraron normalidad multivariante (el índice estandarizado de Mardia obtuvo un valor de $7.129 \mathrm{pa}-$ ra el CVT y de 9.317 para el CVT-P), se aplicó el método de estimación de mínimos cuadrados no ponderados. Los índices de ajuste obtenidos para el CVT fueron los siguientes: $\chi^{2} / \mathrm{gl}=1.667, \mathrm{GFI}=.984, \mathrm{AGFI}=.978, \mathrm{NFI}=.974 \mathrm{y} \mathrm{el}$ SRMR obtuvo un valor de .0562. Las cargas factoriales estandarizadas oscilaron entre .374 y .753 (ver Tabla 2). Paralelamente, el AFC realizado con los datos obtenidos del CVT$\mathrm{P}$ dio lugar a los siguientes índices de ajuste: $\chi^{2} / \mathrm{gl}=1.459$, GFI $=.986$, AGFI $=.980$, NFI $=.980$, obteniendo un SRMR $=.0630$. Como se puede observar en la Tabla 2, las cargas factoriales estandarizadas fueron elevadas, oscilando entre .459 y .790 . En consecuencia, ambos cuestionarios mostraron un buen ajuste al modelo unifactorial propuesto, así como unas adecuadas cargas factoriales estandarizadas.

\section{Relación con otras variables}

El análisis de la posible influencia de determinadas variables sociodemográficas en el nivel de VT de los participantes de este estudio puso de manifiesto que efectivamente sí existe un efecto del género en las puntuaciones obtenidas en la CVT. Los resultados demuestran que las mujeres de esta 
muestra obtienen mayores puntuaciones en lo que la VT se refiere $\left(t_{(192)}=2.869, p<.01\right)$, obteniéndose un tamaño del efecto moderado $(d=.532)$. Paralelamente, no se encontró relación entre la VT y la edad $(r=.10, p=.164)$, ni entre el hecho de estar trabajando o no $\left(t_{191.74)}=1.825, p=.07\right)$.

Tabla 2. Cargas factoriales estandarizadas.

\begin{tabular}{llc} 
& CVT & CVT-P \\
\hline VT1 & .422 & .459 \\
VT2 & .581 & .642 \\
VT3 & .650 & .737 \\
VT5 & .585 & .761 \\
VT6 & .617 & .632 \\
VT7 & .623 & .500 \\
VT8 & .656 & .680 \\
VT9 & .670 & .714 \\
VT10 & .692 & .702 \\
VT11 & .677 & .774 \\
VT12 & .675 & .669 \\
VT14 & .481 & .786 \\
VT15 & .753 & .790 \\
VT16 & .374 & .653 \\
\hline
\end{tabular}

El análisis de la relación entre el CVT con otras variables aporta también evidencias de validez de la escala. Teniendo en cuenta que se obtuvieron diferencias significativas en el nivel de VT de hombres y mujeres, los presentes análisis se llevaron a cabo considerando el género de los participantes.

En primer lugar se analizó la relación entre el CVT y la percepción que los estudiantes acerca de su propia voluntad de trabajo. Se obtuvieron correlaciones estadísticamente significativas tanto en el caso de las mujeres $(r=.583, p<$ $.0001)$ como en el de los hombres $(r=.376, p<.05)$.

En segundo lugar se llevó a cabo un análisis de correlaciones entre la CVT y la subescala de Perseverancia del cuestionario BFQ para estudiar si efectivamente están midiendo constructos similares. Se obtuvieron correlaciones significativas para ambos géneros $(r=.618, p<.0001$ en el caso de las mujeres y $r=.464, p<.01$ en el de los hombres).

Finalmente, considerando que la VT que expresan los estudiantes puede estar influida por un cierto grado de deseabilidad social, se correlacionó el CVT con la subescala de Distorsión del cuestionario BFQ. A diferencia de lo que cabría esperar, la correlación fue de muy poca magnitud en el caso de las mujeres $(r=.231, p<.05)$ y nula en el caso de los hombres $(r=-.038, p=.829)$.

\section{Discusión}

El presente estudio presenta la adaptación del cuestionario de Voluntad de Trabajo a una muestra de estudiantes universitarios españoles.

En relación a los análisis psicométricos realizados, destacar que éstos se han llevado a cabo con los datos obtenidos del CVT y CVT-P, ya que ambos cuestionarios están midiendo el mismo constructo. El primero de ellos es un autoinforme, mientras que el segundo tiene por objetivo evaluar la percepción que tienen los alumnos acerca de la voluntad de trabajo de sus compañeros.

Después de realizar el pertinente análisis de ítems se llegó a la conclusión de que dos de los ítems de la escala debían eliminarse. Ambos ítems tampoco presentaron un óptimo funcionamiento en la versión argentina; no obstante, los autores de la prueba original justificaron su inclusión con el fin de efectuar un análisis cualitativo de los mismos. Los ítems remiten a una modalidad específica de abordaje de las tareas a realizar, evaluando la preferencia de los individuos por enfocarse en varias actividades o especializarse en algún tema.

Una vez depurado el cuestionario se llevó a cabo el análisis de la consistencia interna, obteniendo unas correlaciones ítem-total corregidas adecuadas, así como un índice alpha de Cronbach adecuado para ambos cuestionarios. Asimismo, estas escalas mostraron un buen ajuste al modelo unifactorial propuesto, mediante la aplicación del AFC. Se ha conseguido operacionalizar la VT mediante dos escalas unidimensionales compuestas por 14 ítems cada una. Los aspectos de la VT a los que atañen los ítems son: la disposición al esfuerzo, la capacidad de automotivación frente a las obligaciones y los propios proyectos, el empeño en cumplir con las obligaciones aun contrariando el propio gusto, la inclinación al facilismo, el abocarse con prontitud al trabajo y desarrollarlo con continuidad y sin postergaciones.

El análisis de la relación de la medida de VT con otras variables pone de manifiesto un efecto del género en la medida CVT, demostrando que son las mujeres las que obtienen mayores puntuaciones. Es por ello que el análisis de la relación de la VT con otras variables se ha llevado a cabo separadamente para hombres y mujeres. Paralelamente, y como cabía esperar por su semejanza teórica, se obtuvo una correlación positiva con la escala de Perseverancia del cuestionario BFQ para ambos sexos. Aun así, Abal et al., (2007) describieron diferencias conceptuales entre ambos constructos que justifican que la intensidad de la correlación hallada sea moderada en lugar de elevada. Aunque ambas pruebas son similares en cómo caracterizan a las personas con altas puntuaciones, discrepan en la interpretación de los niveles bajos del rasgo. La escala de Perseverancia permite describir con bajos puntajes a los individuos poco constantes y que faltan a sus compromisos. En cambio, la definición conceptual de VT amplía el espectro de conductas del polo inferior incorporando características como la tendencia al facilismo (i.e. a conformarse con el camino más fácil) o la evitación de tareas que demandan un gran esfuerzo. A su vez, otra distinción importante entre ambos constructos reside en que, a diferencia de la escala Perseverancia, la VT evalúa la percepción del individuo sobre su capacidad para realizar una tarea que no le gusta. La realización de una tarea desagradable supone la existencia constante de tendencias alternativas a la intención que puedan hacer que el individuo posponga el inicio de la tarea, se desvíe de su objetivo o postergue la culminación. 
Sin embargo, los resultados de este estudio apuntan a la escasa relación entre la VT y la subescala de Distorsión del cuestionario BFQ, lo que indicaría que la voluntad de trabajo que informan tener los estudiantes de esta muestra no estaría influida por la deseabilidad social.

Finalmente, destacar las limitaciones del presente estudio. La primera de ellas se refiere a las características de la muestra. La evaluación de la VT es especialmente interesante en el ámbito educativo, lo que a su vez conlleva una serie de limitaciones en relación a las características de la muestra. Es por ello que sería interesante extender el estudio de la VT a estudiantes de otros ámbitos, tanto universitarios como de educación secundaria y formación profesional. La segunda de las limitaciones se refiere a la propia naturaleza del CVT. $\mathrm{Al}$ tratarse de un autoinforme disponemos de la autoevaluación subjetiva de los estudiantes, por lo que el nivel real de VT de cada estudiante puede estar influido por el sesgo de la propia evaluación.

En conclusión, el presente estudio pone de manifiesto las adecuadas propiedades psicométricas de los cuestionarios

\section{Referencias}

Abal, F., Lozzia, G., Aguerri, M. E., Attorresi, H. y Galibert, M. S. (2007). Voluntad de trabajo y percepción de la exigencia académica en estudiantes de psicología. Documento presentado en el $\mathrm{X}$ Congreso de Metodología de las Ciencias Sociales y de la Salud. Barcelona, España.

Abal, F., Lozzia, G., Aguerri, M. E., Galibert, M. S. y Attorresi, H. (2007). Evidencias acerca de la validez de constructo de la escala Voluntad de Trabajo. Anuario de Investigaciones, XV, 283-289.

Alonso Tapia, J. (2005). Motivaciones, expectativas y valores-intereses relacionados con el aprendizaje: el cuestionario MEVA. Psicothema, 17(3), 404-411.

Alonso Tapia, J., Montero, I. y Huertas, J. A. (2000). Evaluación de la motivación en sujetos adultos. El cuestionario MAPE-3. Trabajo no publicado. Registro de Propiedad Intelectual: 91.618. Madrid. Extraído el 15 de octubre de 2010 de http:/ /innova.decp.uam.es/main.php.id=198.

Alonso Tapia, J. y Sánchez Ferrer, J. (1992). El cuestionario MAPE-I: Motivación hacia el aprendizaje. En J. Alonso Tapia (Ed.). Motivar en la adolescencia: Teoría, evaluación e intervención (pp. 53-91). Madrid: Servicio de Publicaciones de la Universidad Autónoma.

Attorresi, H., Abal, F., Lozzia, G., Picón Janeiro, J. Aguerri, M. E. y Galibert, M. S. (2008). Will to Work. Comparison between selfperception and that of the average Argentine. Póster presentado en el III European Congress of Methodology. European Association of Methodology. Oviedo, España.

Bentler, P.M. (2005). EQS 6 Structural equations program manual. Encino, Multivariate Software.

Bentler, P.M. y Bonet, D.G. (1980). Significance tests and goodness of fit in the analysis of covariance structures. Psychological Bulletin, 88, 588-606.

Bermúdez, J. (1995). Manual del Cuestionario "Big Five” (BFQ). Madrid: TEA.

Caprara, G.V., Barbarnelli, C. y Borgogni, L. (1993) Big Five Questionnaie (BFQ). Manuale. Florencia: Organizzazioni Speciali.

Cattell, R.B., Eber, H.W. y Tatsuoka, M.M. (1970) Handbook for the Sixteen Personality Factor Questionaire (16PF). Institute for Personality and Ability Testing, Champaign III.

Costa, P.T. y McCrae, R.R. (1999) Manual NEO PI-R, Inventario de Personalidad NEO Revisado y NEO-FFI, Inventario NEO reducido de Cinco Factores. Madrid: TEA Ediciones, S.A.

Eysenck, H.J. y Eysenck, M. (1985) Personalidad y diferencias individuales. Madrid: Pirámide.

Guilford, J.P. (1975) Factors and factors of personality. Psychological Bulletin, $82(5), 802-814$
CVT y CVT-P para medir la voluntad de trabajo en estudiantes universitarios españoles. Futuras investigaciones tendrán como objetivo ampliar la evidencia de validez convergente con las escalas del MAPE-3 (Alonso Tapia et al., 2000) y el Hakemp-90 (Kuhl, 1994b). Así también, se proyecta aplicar los presentes cuestionarios en otras poblaciones, con el objetivo de evaluar la voluntad de trabajo en otros campos, fuera del ámbito de la educación superior. Tal y como apuntan Attorressi et al. (2008), sería también interesante aplicar los cuestionarios CVT y CVT-P a la población general, lo que nos proporcionaría una visión más amplia acerca del constructo de VT.

Agradecimientos.- Este trabajo se ha llevado a cabo, en parte, gracias a la financiación de la Generalitat de Catalunya (proyecto 2009SGR00822), así como gracias a los subsidios de la Universidad de Buenos Aires (UBACyT P043) y de la Agencia Nacional de Promoción Científica y Tecnológica de Argentina (ANPCyT PICT 20909).

Hu, L.T. y Bentler, P.M. (1999). Cutoff criteria for fit indexes in covariance structure analysis: Conventional criteria versus new alternatives. Struc tural Equation Modeling, 6, 1-55.

Jöreskog, K.F. y Sörbom, D. (1986). LISREL VI: Analysis of linear structural relationships by maximum likelihood, instrumental variables, and least square methods. Morresville, Scientific Software Inc.

Kuhl, J. (1985). Volitional mediators of cognition-behavior consistency: Selfregulatory processes and action versus state orientation. En J.Kuhl y J. Beckman (Eds.), Action control: From cognition to behavior. Nueva York: Springer-Verlag.

Kuhl, J. (1994a). A theory of action and state orientations. En J. Kuhl y J. Beckmann (Eds.), Volition and personality: Action versus state orientation. Seattle: Hogrefe y Huber.

Kuhl, J. (1994b). Action versus state orientation: psychometric properties of the action control scale. En J. Kuhl y J. Beckmann (eds.): Volition and personality: action versus state orientation (47-59). Seattle: Hogrefe y Huber.

Lozzia, G., Abal, F., Aguerri, M. E., Galibert, M. S. y Attorresi, H. (2007) Delimitación del constructo Voluntad de Trabajo. Summa Psicológica UST, 4, 137-148.

Lozzia, G., Abal, F., Blum, G., Aguerri, M., Galibert, M. y Attorresi, H. (2008). Percepción de Docentes y Estudiantes acerca del nivel de Exigencia Académica y Voluntad de Trabajo de los alumnos de Psicología. Memorias del I Encuentro de Docentes e Investigadores de Estadística en Psicología, 121 - 124. Facultad de Psicología, UBA.

Mardia, K.V. (1970) Measures of multivariate skewness and kurtosis with applications. Biometrika, 57, 519-530.

Montero, I. y Alonso Tapia, J. (1992) El cuestionario MAPE-II. En J. Alonso Tapia (Ed.). Motivar en la adolescencia: Teoría, evaluación e intervención (pp. 205-231). Madrid: Servicio de Publicaciones de la Universidad Autónoma.

Moreno-Jiménez, B. (2007). Psicología de la personalidad. Procesos. México: Thomson.

Nunnally, J.C. y Bernstein, I.H. (1994). Psychometric theory (3 ${ }^{\text {rd }}$ edition). New York, McGraw-Hill.

Zuckerman, M. (2002) Zuckerman-Kuhlman personality questionnaire (ZKPQ): an alternative five-factorial model. En B. De Raad y M. Perugini (Eds.), Big five assessment. Seattle: Hogrefe and Huber Publishers.

(Artículo recibido: 31-3-2011, revisado: 4-05-2012, aceptado: 19-02-2012) 


\title{
Anexo 1. Cuestionario de Voluntad de Trabajo (CVT)
}

Marque con una cruz la opción que representa más aproximadamente la frecuencia con la que se presentan en usted las características enunciadas más abajo.

1.- Prefiero aquellas actividades que no demandan mucho esfuerzo.

2.- Necesito que alguien me exija para cumplir bien con mis deberes.

3.- Me esmero para lograr mejores resultados aunque tenga que poner mayor esfuerzo.

4.- Prefiero dedicarme a varias actividades que a pocas.

5.- Me ocupo más de terminar el trabajo cuanto antes que de hacerlo bien.

6.- Aunque me sienta cansado/a termino con la tarea que me impuse.

7.- Doy muchas vueltas antes de ponerme a trabajar.

8.- Realizo un esfuerzo persistente hasta lograr lo que deseo.

9.- Dejo las cosas a medias.

10.- Pongo empeño en cumplir con mis obligaciones incluso cuando no me gustan.

11.- Cualquier excusa me viene bien para interrumpir el trabajo.

12.- Suelo dejar para más tarde el cumplimiento de mis obligaciones.

13.- Prefiero saber un poco de distintos temas que mucho de algunos pocos.

14.- Soy cuidadoso/a en los detalles al realizar una tarea.

15.- Aunque surjan obstáculos, me empeño en alcanzar mis objetivos.

16.- Cuando iba a la escuela, estudiaba como máximo lo imprescindible para aprobar.

\section{Anexo 2. Cuestionario de Voluntad de Trabajo - pares (CVT-P)}

\author{
Marque con una cruz la opción que representa más aproximadamente la frecuencia con la que \\ se presentan en sus compañeros/as de Psicología las características enunciadas más abajo. \\ 1.- Prefieren aquellas actividades que no demandan mucho esfuerzo. \\ 2.- Necesitan que alguien les exija para cumplir bien con sus deberes. \\ 3.- Se esmeran para lograr mejores resultados aunque tengan que poner mayor esfuerzo. \\ 4.- Prefieren dedicarse a varias actividades que a pocas. \\ 5.- Se ocupan más de terminar el trabajo cuanto antes que de hacerlo bien. \\ 6.- Aunque se sientan cansados terminan con la tarea que se impusieron. \\ 7.- Dan muchas vueltas antes de ponerse a trabajar. \\ 8.- Realizan un esfuerzo persistente hasta lograr lo que desean. \\ 9.- Dejan las cosas a medias. \\ 10.- Ponen empeño en cumplir con sus obligaciones incluso cuando no les gustan. \\ 11.- Cualquier excusa les viene bien para interrumpir el trabajo. \\ 12.- Suelen dejar para más tarde el cumplimiento de sus obligaciones. \\ 13.- Prefieren saber un poco de distintos temas que mucho de algunos pocos. \\ 14.- Son cuidadosos en los detalles al realizar una tarea. \\ 15.- Aunque surjan obstáculos, se empeñan en alcanzar sus objetivos. \\ 16.- Están acostumbrados a estudiar como máximo lo imprescindible para aprobar
}

